\title{
Social and Emotional Intelligence (SEI) in BDSM
}

\author{
Eli Pliskin \\ pliskin.eli@gmail.com
}

\section{Introduction}

This article argues that emotional intelligence (EI), also called social and emotional intelligence (SEI) (Goleman, 2006), is central to Bondage and Discipline, Dominance and Submission, and Sadomasochism (BDSM). This paper will use a four-quadrant model of SEI developed by Goleman and colleagues (2002) to illustrate how SEI skills are utilized in BDSM, resulting in durable benefits for those who practice them. This article suggests that BDSM practitioners could use the four-quadrant model as a tool in pursuit of more fulfilling BDSM and life experiences.

\section{Literature Review}

By labeling sadomasochism “perversion,” 19th century psychologists like Krafft-Ebing (1965, p. 25) and Freud (1938, p. 30) contributed to misunderstanding and discrimination that healthy adults practicing BDSM with care and consent have experienced ever since (Williams et al., 2017). When Williams and colleagues (2017) summarized recent literature reviews, they found that, in contrast to early depictions, there is little evidence of an association between BDSM and pathology, and instead there is evidence of beneficial skills and psychological attributes associated with BDSM. This article will examine those benefits through the lens of EI. One central perspective to understand those benefits is that of serious leisure. Drawing on four years of ethnography in one BDSM community, Newmahr (2010a) illustrated how practitioners met three criteria for serious leisure developed by Stebbins (1982): practitioners 1) built a “leisure career," which involved 2) investing effort into developing “specialized skills,” and 3) experiencing “durable benefits" as a result (p. 318).

“Specialized skills” in BDSM include EI skills because subjective experience and relationship are central to BDSM. An online survey of 343 people who reported practicing BDSM indicated that the relationship between partners is fundamental (Faccio et al., 2014). Challenging understandings of pain as exclusively physical and negative, Newmahr (2010b) framed pain in BDSM as similar to an emotion, which can be understood as an experience constructed within a social context. While some play is more focused on physical and technical aspects than others, Newmahr (2011) argued that play is always collaborative and relational.

The concept of Emotional Intelligence (EI), which has not to my knowledge been linked to BDSM, emerged from psychology. Salovey and Mayer (1990) defined EI as "the ability to monitor one's own and others' feelings and emotions, to discriminate among them and to use this information to guide one's thinking and actions" (p. 189). The contemporary understanding of EI has emerged as part of the "affective revolution" of the past 25 years that has sought to balance previous definitions of intelligence in which cognitive ability dominated. EI is understood to be measurable, distinguishable from other forms of intelligence, individually variable, valuable and learnable (Bracket et al., 2016, p. 514). 


\section{Emotional Intelligence in BDSM}

This article illustrates SEI through a popular four-domain model developed by Goleman and colleagues (2002). This model reflects their contention that while there are many models of EI, they all contain two components: 1) awareness and management of self, and 2) awareness and management of others (Cherniss et al., 2010). This model integrates awareness and management of emotion with awareness and management of cognitive, physiological and nervous system processes related to emotion and social interaction. The titles of each quadrant in the figures below are taken from the four domains of this model.

This article modifies the model's quadrants. The larger relationship management quadrant illustrates that the interaction between partners is where the skills come together, and the arrows reflect that even though all domains influence each other, social interactions (relationship management) build on both social awareness and self-management (Bracket et al., 2016), and self-management builds on self-awareness. The figures below list examples of how competencies in each domain are used in negotiating and participating in BDSM play.

As illustrated in the figure below on the left, an individual practices self-awareness in negotiating BDSM play by identifying their wants and limits. As individuals consider routes to fulfill their desires, they may practice self-management in the form of coping with stigma or fear of vulnerability. Once they enter negotiations to play with another person, they practice social awareness by caring about the wants and boundaries of the other person. Finally, they use all the previous competencies in relationship management by negotiating the activities of a scene.

As illustrated in the figure below on the right, individuals practice self-awareness in BDSM play by monitoring themselves from moment to moment. They practice self-management through techniques that aim to regulate their own state. They practice social awareness by tuning into their partners' experiences. Finally, they bring it all together in relationship management in which they communicate with their partner and choose appropriate actions moment to moment to
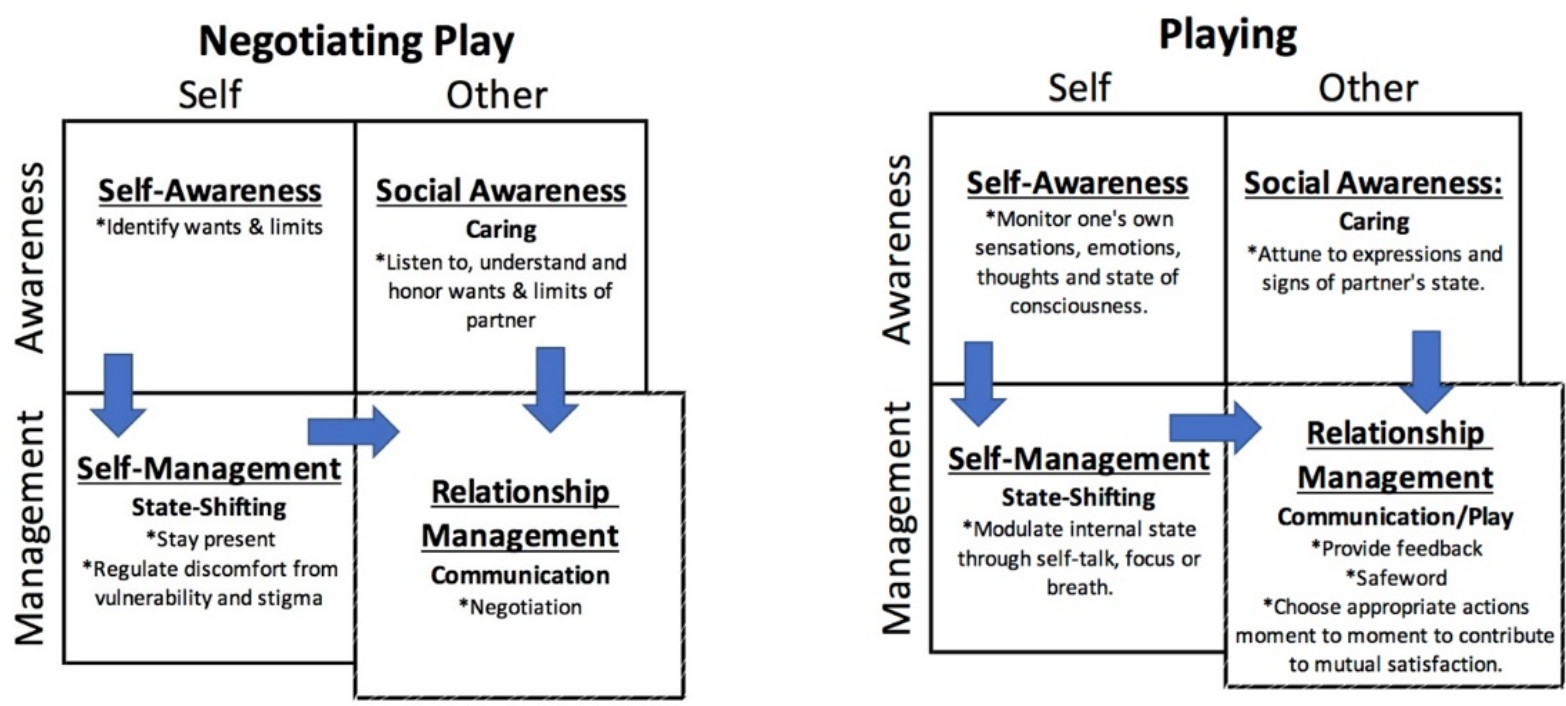
shift states of consciousness and contribute to mutual satisfaction. Three of the four domains will be explored in the following pages to highlight evidence of these EI skills in BDSM.

\section{Self-Management}

EI scholars define self-management to include being comfortable feeling whatever emotions arise, coping with stress and choosing strategies to experience desired states of mind (Bracket et al., 2016). Many struggles, such as losing one's temper, may be mitigated by managing one's emotions. This is achieved through a type of state-shifting, for example, from a stressful state of hyperarousal to a more grounded and present state (Bracket et al., 2016).

Self-management skills recognized by scholars as cultivated in leisure, such as coping with stress (Magnuson, \& Barnett, 2013) and accessing flow (Dieser, 2015), have also been recognized by other scholars as EI capacities. According to Goleman, accessing a state of "flow," which he links to performance at work and fulfillment in life, is an EI capacity. Csikszentmihalyi defined flow (1991) as a pleasurable state of total absorption experienced while using skills to meet meaningful goals. Goleman (2011) emphasized that EI skills of concentration and the ability to cope with stress increase the ability to access flow (Goleman, 2011). State shifting can occur individually or collaboratively. Because of its collaborative nature, state-shifting in BDSM (including reducing stress and accessing flow) will be further examined in the upcoming section on relationship management.

\section{Social Awareness}

Goleman defined three types of empathy as social awareness skills. He distinguished between cognitive empathy (seeing others’ perspectives), emotional empathy (feeling with others) and empathic concern (sensing others' needs, which motivates actions to meet those needs) (Goleman, 2011). This paper considers those three EI capacities to be types of caring.

The 4Cs negotiation framework of Caring, Communication, Caution, and Consent (Williams et al., 2014) emphasizes the importance of caring to BDSM. The framework's originators proposed it in part to emphasize elements of caring and communication, which were not specified in the previous Safe Sane Consensual (SSC) or Risk-Aware Consensual Kink (RACK) frameworks. Williams et al. (2014) argued that caring provides many benefits. It motivates the establishment of alternative communities, "creates safety, trust, and respect for our partners" (p. 6), shapes the "qualitative experiences of BDSM" (p. 3), and "allows us to honor the other as having an equivalent sexual life” (p. 7). If an ethic of care supports responsible BDSM, empathy is part caring, and if empathy is an EI skill, then EI supports ethical BDSM. To realize those benefits, caring in BDSM should include all three types of empathy described by Goleman.

The evolution of the ethic of care parallels the evolution from primarily cognitive models of intelligence to the SEI model. Kohlberg (1971) framed morality as the ability to independently make moral judgements free from the influence of emotion. Gilligan's (1982) feminist ethic of care, in contrast, views moral decisions as subjective and relational, and accounts for multiple ways of understanding (Williams et al., 2014). 
Caring is reflected in the attitudes of BDSM practitioners with regard to oppression. Some feminists have alleged that even consensual sadomasochism prevents practitioners from caring for each other because it is "rooted in patriarchal sexual ideology" (Linden, 1982, p. 4) and necessarily leads to "aggression and the abuse of power" (Linden, 1982, p. 9). Contradicting that claim, a survey revealed that, perhaps as a result of community education that stresses consent, "BDSM practitioners reported significantly lower levels of benevolent sexism, rape myth acceptance, and victim blaming” than “comparison groups” (Klement et al., 2017, p. 130). According to the scale used by the researchers (Glick \& Fiske, 1997), benevolent sexism consists of attitudes that are considered positive by the perceiver but are nonetheless based on stereotypes of women and assumptions of male dominance. Because such assumptions hamper the ability to understand and thus truly care about the perspectives of women, lower levels of benevolent sexism among BDSM practitioners also suggest higher levels of caring.

\section{Relationship Management}

BDSM practitioners engage in the SEI capacity of relationship management by collaborating between partners to shift into flow and other pleasurable, altered states. Ambler et al. (2017) measured 14 participants before and after engaging in BDSM play (a "scene”) and observed evidence of role-specific changes in consciousness. "Tops" (those performing actions on "bottoms" who receive them) exhibited signs of flow, and "bottoms" exhibited signs of a state called transient hypofrontality. Dietrich (2003) hypothesized transient hypofrontality as a neurofunctional commonality between a variety of pleasurable altered states defined by a temporary decrease of activity in the frontal lobe of the brain. Researchers (Ambler et al., 2017, p. 3) explained how their results begin to explain the physiological basis of states described by practitioners as "sub space” or "top space.”

Another way BDSM practitioners collaboratively practice state-shifting is by reducing stress. On average, subjects in the study described above (Ambler et al., 2017) showed decreases in self-reported stress levels from before engaging in a scene to immediately after. Not surprisingly, practitioners describe BDSM play as "playful," and "fun” (Hébert \& Weaver, 2015) and research suggests that play reduces stress (Magnuson, \& Barnett, 2013). Finally, 91.4\% of BDSM practitioners surveyed $(\mathrm{N}=935)$ reported associating BDSM with relaxation or decreased stress most or nearly all of the time (Williams et al., 2016).

There is evidence that BDSM also connects partners and contributes to enjoyment and self-exploration. In addition to decreases in psychological stress, subjects in the previously mentioned psychological study (Ambler et al, 2017) reported increased sexual arousal and connection to each other. In the aforementioned survey (2016) by Williams and colleagues, nearly every respondent reported associating pleasure/enjoyment and positive emotions with BDSM most or nearly all of the time, while self-expression/exploration and adventure are each reported by over $90 \%$.

In addition to state-shifting, Goleman identified communication as a relationship management EI skill. According to Goleman (1995), an important part of communication as an EI capacity is nonverbal. He argued that reading the emotional cues of others requires empathy 
and that there is a relationship between understanding one's own emotions and understanding those of others.

The emphasis on communication skills within the BDSM community further demonstrates the role of EI within BDSM. Kaak (2016) summarized numerous scholarly and community-based authors that cite the centrality of communication to BDSM. Supporting the strengths-based claim that skills cultivated in BDSM could be applied to other areas of life (Williams et al., 2017), individuals with disabilities attributed greater satisfaction in intimate relationships to communication skills learned through BDSM (Kattari, 2015). Because it is impossible to capture all details of play in words, expressing care by taking the effort to understand a partner's perspective (Williams et al., 2016) and reading nonverbal cues are important for mutually beneficial BDSM experiences.

\section{Durable Benefits}

Research demonstrates an association between higher levels of EI and a variety of desirable outcomes including good health, relationship harmony, academic achievement and professional performance (Bracket et al., 2016). With substantial emerging evidence that EI can be developed, doing so has been embraced as a goal by a variety of organizations, schools (from elementary to graduate business programs) and individuals who consume best-selling books on the topic (Bracket et al., 2016). Mental health professionals teach clients EI skills to help them meet a variety of challenges ranging from managing intimate relationships and parenting (Greenberg, 2015) to coping with HIV/AIDS (Johnson \& Naidoo, 2017).

The practice of mindfulness meditation further illustrates how cultivating EI could lead to durable benefits. Goleman identified mindfulness practice as a way to develop EI (Goleman \& Lippincott, 2017). The definition of mindfulness emphasizes that skillful state-shifting is not merely about avoiding discomfort and pursuing pleasure. Mindfulness involves shifting from various default states to attending deliberately in the present to thoughts, feelings and sensations non-judgmentally as they arise and pass (Kabat-Zinn, 1994). The title of a book Goleman wrote with Davidson (2017) illustrated their belief that mastering state-shifting through meditation leads to durable benefits: Altered Traits: Science Reveals How Meditation Changes Your Mind, Brain, and Body (Goleman \& Davidson, 2017). Engaging altered states skillfully, therefore, can contribute to beneficial altered traits.

Harrington (2009) illustrated in Sacred Kink that many BDSM practitioners deliberately strive for experiences of transcendence and durable benefits such as personal growth:

By consciously engaging in altered states of consciousness instead of letting them appear in our lives when we trance out while driving or happen to fall into sub space on accident, we become psychonauts. Literally translated from Greek as a "sailor of the soul," a psychonaut has the power to investigate their own minds, bodies, and spirits through stepping sideways and looking at themselves through a new lens... by engaging in the use of tools as old as humankind's quest for altered states, we can come to the same results as our ancestors-visions, dreams, truths, and a life transformed. By breaking the 
boundaries of ordinary reality through our exploration of kink, we have the power to step out of 'normal' and into the altered, the extraordinary. (p. 19)

While early psychologists associated deviating from normality with disorder, Harrington (2009) associated transcending the normal with the "extraordinary." By emphasizing "a life transformed," he showed similarities between BDSM activities and spiritual practices in which participants achieve what Goleman and Davidson (2017) called altered traits as a result of navigating altered states.

A study which found that BDSM practitioners have higher levels of a variety of desirable characteristics indicates that they may enjoy altered traits as a result of their ability to navigate altered states:

BDSM participants, as a group are, compared with non-BDSM participants, less neurotic, more extraverted, more open to new experiences, more conscientious, yet less agreeable.

BDSM participants were also less rejection sensitive, whereas female BDSM participants had more confidence in their relationships, had a lower need for approval, and were less anxiously attached compared with non-BDSM participants. Finally, the subjective wellbeing of BDSM participants was higher than that of the control group. (Wismeijer et al., 2013, p. 7)

\section{Future Directions}

Further investigation would be helpful. Research may explore ways that BDSM practitioners utilize their SEI skills in BDSM to contribute to both momentary enjoyment as well as lasting relationship satisfaction, personal growth and well-being, testing whether there is a relationship between altered states in BDSM and altered traits. It could measure whether SEI is associated with satisfaction in BDSM, explore what accounts for individual SEI variation, and discover how to promote more SEI-related benefits. While this article limited its scope to negotiation and play, similar analyses could be carried out with other aspects of the BDSM lifestyle such as participating in local community, fighting oppression, or developing long-term relationships. Further investigation could also explore how EI could help address challenges faced by practitioners such as stigma (Williams et al., 2017), community conflict (Graham et al., 2016), consent violations (Haviv, 2016) and emotional dips experienced after intensely satisfying play experiences (“sub drop”) (Sprott \& Randall, 2016).

Applying the four-domain SEI model to BDSM can benefit practitioners. Doing so is both descriptive and prescriptive. It recognizes SEI skills that practitioners cultivate and recommends opportunities for deepening satisfaction. The spatial organization of quadrants offers a comprehensive way to understand how BDSM skills relate to each other. The quadrants provide a comprehensive, easy-to-remember, and elegant framework to educate practitioners (and professionals who serve them) about BDSM. While the 4Cs provide a useful framework for negotiation (Williams et al., 2016), the SEI quadrant provides a complementary framework for situating BDSM within a context of personal development. Through the quadrants, practitioners could assess their strengths and areas for improvement within BDSM activities. Using a strengths-based approach (Williams et al., 2017), they could apply SEI skills cultivated in BDSM 
to all areas of life. Finally, looking at BDSM through an SEI lens offers the added benefit of confronting stigma by linking BDSM with a psychological capacity (SEI) already recognized as beneficial.

\section{References}

Ambler, J., Lee, E., Klement, K., Loewald, T., Comber, E., Hanson, S., \& Sagarin, B. (2017). Consensual BDSM facilitates rolespecific altered states of consciousness: A preliminary study. Psychology of Consciousness: Theory, Research, and Practice, 4(1), 75-91.

Bracket, M. A., Rivers, S. E., Bertoli, M. C. \& Salovey, P. (2016). Emotional Intelligence. In L. Feldman Barrett, M. Lewis, \& J. M. Haviland-Jones (Eds.), Handbook of emotions (4 ${ }^{\text {th }}$ ed., pp. 513-531). New York, NY: The Guilford Press.

Cherniss, C, Extein, M, Goleman, D. \& Weissberg, R. P. (2010). Emotional Intelligence: What does the research really indicate? Educational Psychologist, 41, 239-245.

Csikszentmihalyi, M. (1991). Flow: The psychology of optimal experience. New York, NY: Harper Collins.

Dieser, R. B., Christenson, J., \& Davis-Gage, D. (2015). Integrating flow theory and the serious leisure perspective into mental health counseling. Counselling Psychology Quarterly, 28(1), 97-111.

Dietrich, A. (2003). Functional neuroanatomy of altered states of consciousness: The transient hypofrontality hypothesis. Consciousness and Cognition, 12, 231-256.

Faccio, E., Casini, C., \& Cipolletta, S. (2014). Forbidden games: The construction of sexuality and sexual pleasure by BDSM 'players.' Culture, Health \& Sexuality, 16(7), 752-764.

Freud, S. (1938). Sadism and masochism. In T. Weinberg \& G. W. L. Kamel (Eds.) (1983) S and $M$ : New studies in sadomasochism (pp. 25-29). Buffalo, NY: Prometheus Books.

Gilligan, C. (1982). In a different voice. Cambridge, MA: Harvard University Press.

Glick, P., \& Fiske, S. T. (1997). Hostile and Benevolent Sexism: Measuring Ambivalent Sexist Attitudes Toward Women. Psychology of Women Quarterly, 21(1), 119-135.

Goleman, D. (1995). Emotional Intelligence: Why it can matter more than IQ. New York, NY: Bantam Books.
Goleman, D. (2006). Social intelligence: The new science of human relationships. New York, NY: Bantam Dell.

Goleman, D. (2011) The brain and emotional intelligence: New insights. Florence, MA: More Than Sound.

Goleman, D., \& Davidson, R. J. (2017). Altered traits: Science reveals how meditation changes your mind, brain, and body. New York, NY: Avery.

Goleman, D. \& Lippincott, L. (2017, September 8). Without Emotional Intelligence, mindfulness doesn't work. Harvard Business Review. Retrieved from https://hbr.org/2017/09/sgc-what-reallymakes-mindfulness-work

Graham, B. C., Butler, S. E., McGraw, R., Cannes, S. M., \& Smith, J. (2016). Member perspectives on the role of BDSM communities. The Journal of Sex Research, 53(8), 895-909.

Greenberg, L. S. (2015). Emotion-focused therapy: Coaching clients to work through their feelings (2nd ed.). Washington, DC: American Psychological Association.

Harrington, L. (2009). Sacred kink: The eightfold path of BDSM and beyond. Morville, NC: Lulu Enterprises.

Haviv, N. (2016). Reporting sexual assaults to the police: The Israeli BDSM community. Sexuality Research \& Social Policy, 13(3), 276-287.

Hébert, A. \& Weaver, A. (2015). Perks, problems, and the people who play: A qualitative exploration of dominant and submissive BDSM roles. The Canadian Journal of Human Sexuality, 24(1), 49-62.

Johnson, S. M., \& Naidoo, A. V. (2017). A psychoeducational approach for prevention of burnout among teachers dealing with HIV/AIDS in South Africa. AIDS Care, 29(1), 73-78.

Kaak, A. (2016) Conversational phases in BDSM pre-scene negotiations. Journal of Positive Sexuality, 2(3), 47-52.

Kabat-Zinn, J. (1994). Wherever you go, there you are. New York, NY: Hyperion. 
Kattari, S. (2015). “Getting it”: Identity and sexual communication for sexual and gender minorities with physical disabilities. Sexuality \& Culture, 19 (4) 882-899.

Klement, K. R., Sagarin, B. J., \& Lee, E. M. (2017). Participating in a culture of consent may be associated with lower rape-supportive beliefs. Journal of Sex Research, 54(1), 130-134.

Kohlberg, L. (1971). From is to ought: How to commit the naturalistic fallacy and get away with it in the study of moral development. In T. Mischel (Ed.), Cognitive development and epistemology (pp. 151-235). New York, NY: Academic Press.

Krafft-Ebing, R. (1965). Psyhchopathia sexualis. In T. Weinberg \& G. W. L. Kamel (Eds.). (1983) $S$ and M: New studies in sadomasochism (pp. 25-29). Buffalo, NY: Prometheus Books.

Linden, R. R. (1982). Introduction: Against sadomasochism. In R. R. Linden, D. R. Pagano \& S. L. Star (Eds.) Against sadomasochism: a radical feminist analysis (pp. 1-15). East Palo Alto, CA: Frog in the Well.

Magnuson, C. D., \& Barnett, L. A. (2013). The playful advantage: How playfulness enhances coping with stress. Leisure Sciences, 35(2), 129-144.

Newmahr, S. (2010a). Rethinking kink: Sadomasochism as serious leisure. Qualitative Sociology, 33(3), 313-331.

Newmahr, S. (2010b). Power struggles: Pain and authenticity in SM play. Symbolic Interaction, 33(3), 389-411.
Newmahr, S. (2011). Chaos, order, and collaboration: Toward a feminist conceptualization of edgework. Journal of Contemporary Ethnography, 40(6), 682-712.

Salovey, P. \& Mayer, J.D. (1990). Emotional Intelligence. Imagination, Cognition and Personality, 9(3), 185-211.

Sprott, R. \& Randall, A. (2016). Black and blues: Sub drop, top drop, event drop and scene drop. Journal of Positive Sexuality, 2(3), 53-61.

Stebbins, R. A. 1982. Serious leisure: A conceptual statement. Pacific Sociological Review 25(2), 251-72.

Williams, D J, Prior, E. E., Alvarado, T., Thomas, J. N., \& Christensen, M. C. (2016). Is Bondage and Discipline, Dominance and Submission, and Sadomasochism recreational leisure? A descriptive exploratory investigation. The Journal of Sexual Medicine, 13(7), 10911094.

Williams, D J, Thomas, J., Prior, E., Amezquita, C. \& Hall, D. (2017). Social work practice with clients that enjoy participation in consensual BDSM: Identifying and applying strengths. Journal of Positive Sexuality, 3(1), 12-20.

Williams, D J, Thomas, J. N., Prior, E. E., \& Christensen, M. C. (2014). From "SSC" and "RACK" to the "4Cs": Introducing a new framework for negotiating BDSM participation. Electronic Journal of Human Sexuality, 17, Retrieved from http://www.ejhs.org/volume17/BDSM.html

Wismeijer, A. A., \& van Assen, M. A. (2013) Psychological Characteristics of BDSM Practitioners. The Journal of Sexual Medicine, 10(8), 1943-1952. 